\title{
R5B. ANonymus
}

\section{„Mascaron-Fassung“ (Stintzing 2)}

Processus diaboli contra genus humanum / Tractatus procuratoris sub nomine diaboli / Tractatus quaestionis ventilatae coram domino nostro Jesu Christo inter virginem Mariam ex una parte et diabolum ex alia parte / Tractatus (Libellus) procuratoris in quo diabolus producit litem coram iudice omnipotente Deo contra genus humanum

Praefatio: Nur zwei Handschriften (Frankfurt und Praha) haben eine Praefatio, die der Praefatio der zwei anderen Fassungen sehr ähnlich ist. Dazu vgl. unter „Überlieferung““.

Incipit: Accessit Mascaron ad Dei omnipotentis praesentiam et ait... [Varianten bei den Namen des Teufels: Mascharon, Ascaron, Ascharon].

Explicit: Das Explicit variiert je nachdem, ob die zwei letzten Sätze vorkommen oder nicht. Es unterscheidet sich nicht nach Fassungen:... remansit caelestis militia cum gaudio [einige Hss. fügen hinzu: ingenti laudans advocatam $] ;. .$. spes nostra salve; ... nullum rite fundatur exordium.

Autor: In der Überlieferung findet sich kein Hinweis auf einen möglichen Autor. Stintzing hat einen spanischen oder französischen Autor vermutet, da der Name des Anwalts der Hölle, Mascaron, in diesen Sprachen interpretierbar wäre. Allerdings ist das Wort mascha, in den Bedeutungen „Hexe“ und „Maske, Fratze“ schon im Frühmittelalter von Italien bis England nachweisbar (siehe die Belege in Du Cange 5, 293). Es wäre darum zu gewagt, den Namen Mascaron allein als Beweis für einen bestimmten Ursprung zu nehmen. Außerdem variiert der Name in der Überlieferung und kommt auch als Ascaron vor. Diese zweite Form nimmt Burdach als ursprünglich an und sieht in ihr eine Ableitung aus Astaroth, einem mehrmals im Alten Testament genannten phönizischen Gott. Diesen Namen führen ebenfalls Teufel in Mysterienspielen und in Piers Plowman (Burdach 488). Eine dritte, mögliche Erklärung des Namens wäre eine Ableitung aus dem biblischen Accaron, dem Kultort des Beelzebub (IV Reg 2-6).

Die Entstehung der Advocacia-Fassung in Cambrai und der niederdeutschen Übersetzung der Mascaron-Fassung in den südlichen Niederlanden weist auf das Gebiet Nordostfrankreich-Belgien-Niederlande als möglichen Aufenthaltsort des Autors hin. 
Datierung: Wenn dieser Text die Vorlage für Jacob van Maerlant war, muss er vor 1262 (Abfassungsjahr des Boek van Merline) entstanden sein. Es ist allerdings auch möglich, dass Maerlant und der unbekannte Autor der Mascaron-Fassung eine für uns verlorene, gemeinsame Quelle hatten. Die Diskussion der vier Anwältinnen in dieser Fassung ist vom Streit der Töchter Gottes inspiriert, ein Motiv aus dem Psalm 84, das vor allem durch eine Predigt Bernhards von Clairvaux, sermo I in Festo Annuntiationis, bekannt wurde (dazu vgl. Mäder, 21-24 und Ohly, 260-277). Sie ist demnach frühestens um die Mitte des 12. Jhs. und spätestens vor dem Ende des 14. Jhs., der Entstehungszeit des ältesten Textzeugen (Hs. in Bologna), entstanden.

Inhalt: Zusammenfassung nach dem Druck H 2647. Diese Fassung hat in der Regel keine Praefatio (Ausnahmen: Handschriften in Frankfurt und Praha, katalanische Übersetzung) und fängt mit dem Auftreten des Anwalts der Hölle an, der Mascaron oder Ascaron genannt wird. Die Handlung entspricht der Advocacia-Fassung bis zu dem Punkt, da der Teufel eine Aufteilung der Menschheit vorschlägt und Maria antwortet, die Schuld der Angeklagten sei durch Christus am Kreuz gesühnt worden. Mascaron verlangt dann die Hilfe zweier Anwältinnen, Justitia und Veritas. Daraufhin beantragt Maria die Hilfe von Misericordia und Pax. Die vier Anwältinnen sind die Töchter Gottes aus dem Psalm 84 (vgl. „Datierung“"). Der Teufel sagt, Lucifer sei bestraft worden und die Gerechtigkeit verlange, dass der Mensch gleich behandelt werde. Maria antwortet, dass der Engel keinen Makel in seiner Natur hatte, der Mensch sei aber schwach. Der Teufel erwidert, Adam und Eva haben ein ausdrückliches Gebot missachtet. Maria antwortet, dass der Engel keines Gebotes bedurfte, weil er es von Natur aus kannte; dass der Mensch schwach sei und dass eine Bestrafung die Schöpfung der Menschheit sinnlos machen würde. Justitia und Veritas ergreifen das Wort, um Mascaron zu unterstützen. Anschließend redet Misericordia und kann Gottvater überzeugen. Pax vermittelt: Justitia hatte eine unendliche Strafe für eine unendliche Sünde beantragt, und eben dies sei durch Jesu Tod am Kreuz geschehen. Die vier Töchter Gottes küssen und versöhnen sich, Mascaron zieht sich zurück und die Engelchöre singen Salve regina.

Editionen: Unediert.

\section{Übersetzungen / Bearbeitungen:}

Ins Mittelniederdeutsche als Episode (cap. X-XII, v. 1694-2981) im Boek van Merline des Jacob van Maerlant, entstanden 1262. 
Sodmann, Timothy (Hg.), Jacob van Maerlant. Historie van den Grale und Boek van Merline, Köln / Wien 1980.

Ebenfalls ins Mittelniederdeutsche als selbständiges Gedicht mit dem Titel „Dit es van Maskeroen“:

Snellaert, Ferdinand A., Nederlandsche Gedichten uit de veertiende eeuw van Fan Boendale, Hein van Aken en anderen naar het Oxfordsch Handschrift, Brussel 1869, LXII-LXXVIII und 493-538.

Ins Katalanische im 14. Jh.:

Bofarull y Mascaró, Pascual de, Documentos literarios en antigua lengua catalana (siglos XIV y XV), Barcelona 1857, 107-117. Der Herausgeber gibt als Quellen zwei Codices an, den einen in S. Cucufat del Vallés mit dem Titel Miscellanea ascetica, den anderen in Santa María de Ripoll, 155, die er ohne weitere Informationen Ende des 14., Anfang des 15. Jhs. datiert. Er bietet einen Text ohne Apparat an. Im Gegensatz zur lateinischen Fassung hat dieser Text eine Praefatio, die jener der Advocacia-Fassung stark ähnelt.

$\mathrm{Zu}$ den Übersetzungen ins Mittelniederdeutsche und ins Katalanische:

Crawford, J. Wickersham, ,The Catalan Mascarón and an Episode in Jacob van Maerlant's Merlin", Publications of the Modern Language Association of America 26 = N.S. 19, (1911), 31-50.

Folgender Text wird von Norbert Ott (Rechtspraxis und Heilsgeschichte. Zu Überlieferung, Ikonographie und Gebrauchssituation des deutschen Belial, München 1983, 6 Anm. 9) als Bearbeitung des Belial und des Processus erwähnt, es handelt sich jedoch um eine Bearbeitung des Belial:

Jacob Ayrer, Historischer Processus iuris. In welchem sich Lucifer uber Fesum darumb daß er ihme die hellen zerstört, eingenommen, die gefangene daraus erlöst und hingegen ihnen Lucifern gefangen und gebunden habe auff das aller heffigest beklaget, Frankfurt a. M.: Nicolaus Bassaeus, 1597 (häufig nachgedruckt, zu diesem Text Stintzing, 278).

\section{Literatur:}

Mäder, Eduard Johann, Der Streit der Töchter Gottes. Zur Geschichte eines allegorischen Motivs, Bern / Frankfurt a. M. 1971, 21-24 und 40-45.

Ohly, Friedrich, „Die Trinität berät über die Erschaffung des Menschen und über seine Erlösung“", Beiträge zur Geschichte der Deutschen Sprache und Literatur 116 (1994), 242-284 (Ohly berücksichtigt nicht diesen Text).

\section{Überlieferung:}

Die verwendeten Kürzel sind aufgeschlüsselt unter „Überlieferung (für die drei Fassungen)".

Handschriften:

Berlin, SBB-PK, lat. fol. 628, a. 1439, n³ 3. Kolophon:... facta per episcopum Corbucen (Dolezalek, Bd. 1).

Bologna, Collegio di Spagna, 126, Ende des 14. Jhs., fol. 189-195. Ohne Titel (In principio).

Eichstätt, UB, Cod. st 417, 15. Jh., fol. 364. Ohne Titel (In principio).

Escorial, Real Biblioteca de San Lorenzo, lat. d. II. 12, 15. Jh., fol. 249r-253v (García y García, 19). 
Frankfurt a. M., StUB, Ms. Praed. 125, 15. Jh. (Mitte), fol. 169r-175r, incipit: Nostis fratres carissimi quod cum filius dei perambularet totam terram demones eum multipharie temptauerunt..., in dieser Handschrift geht dem Text eine Praefatio voraus, die mit der Praefatio in der Bartolo-Fassung große Ähnlichkeit aufweist. Der Text bricht am Anfang der Rede der Justitia ab, mit den Worten... Qui cum facit peccatum servus est peccati. Rursum si homo nunquam peccasset. Konsultiert in Reproduktionen (Reader-Printer-Kopien) (MssMed).

Klosterneuburg, StiftsB, 637B, $\mathrm{n}^{\circ}$ 7, Jh.?, fol.? (In principio).

Leiden, BR, Ablaing 29, a. 1467, fol. 157-162r. Titel: Processus diaboli contra genus humanum (Calasso).

München, BSB, Clm 3331, a. 1502, fol. 201r-214r (Walther, dort irrtümlich als Clm 337 angegeben). Titel: Processus iudiciarius, Litigatio Mascaron contra genus humanum. Der Text dieser Handschrift bietet genaue Hinweise auf das kanonische und das zivile Recht wie die Bartolo-Fassung. Sie wurden durch rote Unterstreichung kenntlich gemacht. Autopsie.

München, UB, $2^{\circ}$ 301, 15. Jh., fol. 116r-119r. Titel: Tractatus procuratoris editus sub nomine dyaboli quando petiit iusticiam coram Deo et beata Maria Virgo se opposuit contra ipsum et obtinuit. Autopsie (Casamassima, 139).

Praha, NK, VI. A. 5 (Truhlář 1016), a. 1407, fol. 1ra-5rb. Wie die Frankfurter Handschrift bietet diese Handschrift eine Praefatio, die der Praefatio der zwei anderen Redaktionen nahe steht. Dazu Burdach, 489-490, mit Abdruck des Textes der Praefatio. Kolophon: Explicit pulcher tractatus qui potest dici consistorium diaboli.

Vaticano, BAV, Vat. lat. 10726, 15. Jh. (Mitte), fol. 121 (In principio).

Würzburg, UB, M. ch. f. 5, 15. Jh., fol. 65v-69r. Titel: Tractatus procuratoris editus sub nomine dyaboli quando petiit iusticiam coram Deo et beata Maria virgo se opposuit contra ipsum et obtinuit (Casamassima, 176).

Frühdrucke:

Tractatus quaestionis ventilatae coram domino nostro fesu Christo inter virginem Mariam ex una parte et diabolum ex alia parte [Augsburg: Günther Zainer, vor 1473]. H 8589, BSB-Ink. B-211.

Tractatus (Libellus) procuratoris in quo dyabolus producit litem coram iudice omnipotente deo contra genus humanum pro quo beata virgo Maria tanquam procuratrix et aduocata comparens tandem pugnam obtinuit et inimici versuciam confudit, Venedig: Gerardus de Flandria (de Lisa), 1478. H 2647, BSB-Ink. B-212.

Tractatus procuratoris editus sub nomine diaboli quando petiit iusticiam coram Deo et beatissima Virgo Maria se opposuit contra ipsum et obtinuit necnon obmutuit pugna contra genus humanum, [Roma: Stephan Plannck, c. 1491-1500]. HCR 2643 (?), BSB-Ink. B-213. 